\title{
Extreme and exposed representing measures of the disk algebra
}

\author{
by Alex Heinis (Leiden) and Jan Wiegerinck (Amsterdam)
}

\begin{abstract}
We study the extreme and exposed points of the convex set consisting of representing measures of the disk algebra, supported in the closed unit disk. A boundary point of this set is shown to be extreme (and even exposed) if its support inside the open unit disk consists of two points that do not lie on the same radius of the disk. If its support inside the unit disk consists of 3 or more points, it is very seldom an extreme point. We also give a necessary condition for extreme points to be exposed and show that so-called BSZ-measures are never exposed.
\end{abstract}

1. Introduction and notational matters. In a previous paper [2] Brummelhuis and the second author studied representing measures for the disk algebra

$$
A=A(D)=\{f \in C(\bar{D}) \mid f \text { is holomorphic on } D\},
$$

where $D$ is the unit disk in $\mathbb{C}$. As usual $A$ is endowed with the structure of a uniform algebra. Its spectrum equals $\bar{D}$. That is, every homomorphism $\phi: A \rightarrow \mathbb{C}$ is of the form $f \mapsto f(x)$ for some $x \in \bar{D}$. The Hahn-Banach theorem allows for a multitude of extensions of $\phi$ as a continuous linear functional on $C(\bar{D})$. The dual space $C(\bar{D})^{*}$ can be identified with $M$, the space of complex Borel measures on $\bar{D}$ endowed with the weak* topology by the Riesz representation theorem. A representing measure $\mu$ for $x \in D$ is a positive Borel measure on $\bar{D}$ such that

$$
\int f d \mu=f(x) \quad \forall f \in A .
$$

So $\mu$ represents some extension of $f \mapsto f(x)(f \in A)$ to $C(\bar{D})$. Setting $f=1$, we find that $\mu$ is a probability measure. Let $M_{0}$ denote the set of positive representing measures for 0 for $A(D)$. Clearly, $M_{0}$ is a convex and

2000 Mathematics Subject Classification: 46J15, 52A07, 30H05.

Key words and phrases: extreme point, exposed point, representing measure, disk algebra. 
weak*-compact subset of $M$. For the above and for more background in uniform algebras the reader may consult [3].

Let $E$ be a relatively closed subset of $D$ and let $T$ denote the boundary of $D$. By $M_{E}$ we denote the subset of $M_{0}$ of representing measures $\mu$ with $T \subset \operatorname{supp} \mu \subset T \cup E$. Clearly, $M_{E}$ is an extreme set of $M_{0}$, i.e. if a point in $M_{E}$ is an interior point of a line segment in $M_{0}$, this line segment is contained in $M_{E} . M_{0}$ is very large and it is reasonable (cf. [2]) to consider only representing measures $\mu$ with $T \subset \operatorname{supp} \mu$.

The following result was obtained in [2].

TheORem 1.1. Let $\mu$ be an extreme point of $M_{0}$. Suppose that $\mu \in M_{E}$ where $E$ is a compact subset of $D$. Then $E$ is a finite set.

Also, it was shown that for each $z_{0} \in D \backslash\{0\}$ there exists one extreme point $\mu \in M_{0}$ with $\operatorname{supp} \mu=T \cup\left\{z_{0}\right\}$. Recently Izzo [5] showed that these $\mu$ are also exposed in $M_{0}$.

Most of the present paper is concerned with the case when $E$ is finite. We thoroughly investigate the situation where $E$ consists of two points. Then $M_{E}$ is a strictly convex subset of $M_{0}$ if and only if these points are not on a diameter of $D$; moreover, extreme points in $M_{E}$ are exposed.

When $E$ consists of more than 2 points, the situation becomes much more complicated. We give examples of sets $E$ consisting of $n$ points in general position in $D$ that are subsets of the support of an extremal element of $M_{0}$ and examples of sets $E$ for which this is not the case. We present a sufficient condition for extreme points of $M_{E}$ to be exposed and give examples of extreme points of $M_{E}$ that are not exposed.

In passing we show that measures $\mu$ in $M_{D}$ such that $\mu(T)=0$ cannot be exposed. From [2] we know that such measures can be extreme points of $M_{0}$.

Acknowledgments. We are grateful to Erik Hendriksen and Chris Klaassen for their useful remarks on this work.

2. Preliminary observations. The Poisson transform or balayage or sweep of $\nu$ is defined for any finite measure $\nu$ on $D$ as the $L^{1}$-function on $T$ given by

$$
\langle\nu, P(\zeta)\rangle=\int_{D} \frac{1-|z|^{2}}{|\zeta-z|^{2}} d \nu(z) .
$$

Here $P(\zeta)=P(\zeta, z)=\left(1-|z|^{2}\right) /|\zeta-z|^{2}$, the Poisson kernel. The notation is chosen to stress the fact that for fixed $\zeta \in T, P(\zeta)$ may be viewed as a functional on spaces of compactly supported complex Borel measures on $D$. See e.g. [6] or [4] for more details. We recall from [2] the following 
TheOREM 2.1. If $\mu \in M_{0}, \nu=\left.\mu\right|_{D}$ then

$$
\mu=\nu+(1-\langle\nu, P(\zeta)\rangle) \frac{d \theta}{2 \pi} \quad\left(\zeta=e^{i \theta}\right)
$$

and $0 \leq\langle\nu, P(\zeta)\rangle \leq 1$. Conversely, if $\nu$ is a positive Borel measure such that $0 \leq\langle\nu, P(\zeta)\rangle \leq 1$ then the measure defined by (2) is in $M_{0}$.

The function $f=(2 \pi)^{-1}(1-\langle\nu, P(\zeta)\rangle)$ will be referred to as the trace of $\mu, f=\operatorname{Tr} \mu$. For $\mu \in M_{0}$ we will write

$$
\mu=\mu_{0}+\mu_{1}, \quad \mu_{0}=\left.\mu\right|_{D}, \quad \mu_{1}=f \frac{d \theta}{2 \pi},
$$

to indicate the decomposition (2).

From all this it follows that $M_{0} \leftrightarrow\{\nu \in M \mid \nu \geq 0, \nu(T)=0,\langle\nu, P(\zeta)\rangle$ $\leq 1\}$, which describes $M_{0}$ more concretely. Moreover, this shows that even $M_{D}$ is huge. We quote from [2]:

Theorem 2.2. If $\mu \in M_{0}$ is extreme, $T \subset \operatorname{supp} \mu$ and $\operatorname{Tr} \mu$ has a realanalytic minorant $h$ which is $\geq 0$ but not identically 0 , then $E:=\operatorname{supp} \mu \cap D$ is finite.

Corollary 2.3. If $\mu \in M_{E}$ is extreme with $E$ compact, then $E$ is finite.

Indeed, $P_{\nu}$ is clearly real-analytic and if $\operatorname{Tr} \mu \equiv 0$ then $\mu$ would be supported by a compact subset of $D$, contradicting $T \subset \operatorname{supp} \mu$.

The following trivial observations will be used later on.

Lemma 2.4. Let $\mu$ be a positive measure with bounded Poisson transform. Suppose that $\operatorname{supp} \mu \subset\left[0, e^{i \theta_{0}}\right]$. If $\mu$ has support outside 0 , then $\left\langle\mu, P\left(e^{i \theta}\right)\right\rangle$ is decreasing on $\left(\theta_{0}, \theta_{0}+\pi\right)$ and increasing on $\left(\theta_{0}-\pi, \theta_{0}\right)$. Moreover, with $\sigma$ denoting reflection in the line $t e^{i \theta_{0}}$, we have

$$
\langle\mu, P(\zeta)\rangle=\langle\mu, P(\sigma(\zeta))\rangle .
$$

Lemma 2.5. If $\mu(0)>0$ and $\mu$ is extreme then $\mu=\delta_{0}$.

Proof. Suppose that $0<\mu(0)<1$; put $\mu(0)=c, \nu=\mu-c \delta_{0}, \nu^{*}=$ $\nu /(1-c)$. Then $\nu^{*}$ is representing, $\nu^{*} \neq \delta_{0}, 0<c<1$ and $\mu=(1-c) \nu^{*}+c \delta_{0}$, hence $\mu$ is not extremal.

Lemma 2.6. If $E \subset[0,1]$ and $\mu$ is extreme, then $|E| \leq 1$.

Pro of. Suppose there exist two relatively open disjoint sets $U, V \subset E$ of positive $\mu$-measure. Let

$$
\delta_{1}=\left\langle\left.\mu\right|_{U}, P(1)\right\rangle, \quad \delta_{2}=\left\langle\left.\mu\right|_{V}, P(1)\right\rangle, \quad W=D \backslash(U \cup V)
$$

and define

$$
\nu_{t}=\left.\left(1+t \delta_{2}\right) \mu\right|_{U}+\left.\left(1-t \delta_{1}\right) \mu\right|_{V}+\left.\mu\right|_{W}, \quad|t| \leq \frac{1}{\delta_{1}}, \frac{1}{\delta_{2}} .
$$


Then $\nu_{t}$ is a positive measure on $D$ and

$$
\begin{aligned}
\left\langle\nu_{t}, P(\zeta)\right\rangle & \leq\left\langle\nu_{t}, P(1)\right\rangle=\left(1+t \delta_{2}\right) \delta_{1}+\left(1-t \delta_{1}\right) \delta_{2}+\left\langle\left.\mu\right|_{W}, P(1)\right\rangle \\
& =\left(\left\langle\left.\mu\right|_{U}, P\right\rangle+\left\langle\left.\mu\right|_{V}, P\right\rangle+\left\langle\left.\mu\right|_{W}, P\right\rangle\right)(1)=\langle\nu, P(1)\rangle \leq 1
\end{aligned}
$$

hence

$$
\mu_{t}=\nu_{t}+\frac{1}{2 \pi}\left(1-\left\langle\nu_{t}, P(\zeta)\right\rangle\right) d \theta \in M_{0} .
$$

With $|t| \leq 1 / \delta_{1}, 1 / \delta_{2}$ we have $\mu=\mu_{0}=\left(\mu_{t}+\mu_{-t}\right) / 2$ and since $\mu_{t} \neq \mu_{-t}$, we find that $\mu$ is not an extreme point of $M_{0}$.

Notation 2.7. For finite $E$ we will use the following notation. Let $E=\left\{a_{1}, \ldots, a_{n}\right\} \subset D$. With a positive measure $\mu=\sum_{i=1}^{n} c_{i} \delta_{a_{i}}$ on $E$ we associate $c=c(\mu)=\left(c_{1}, \ldots, c_{n}\right) \in \mathbb{R}^{n}$. We write $p_{i}(\theta)=P\left(e^{i \theta}, a_{i}\right), p(\theta):=$ $\left(p_{1}(\theta), \ldots, p_{n}(\theta)\right)$. Then $\langle\mu, P(\zeta)\rangle=\sum_{i=1}^{n} c_{i} P\left(\zeta, a_{i}\right)=:\langle c, p(\theta)\rangle, \zeta=e^{i \theta} \in T$. Let $\Lambda_{E}=\left\{\left(c_{1}, \ldots, c_{n}\right) \in \mathbb{R}^{n} \mid c_{i} \geq 0,\langle c, p(\theta)\rangle \leq 1 \forall \zeta=e^{i \theta} \in T\right\}$.

The set $M_{E}$ is mapped by $c$ isomorphically to $\Lambda_{E}$ and, denoting by $\operatorname{Ex}(A)$ the set of extreme points of a set $A$, we have

$$
\operatorname{Ex}\left(M_{E}\right)=\operatorname{Ex}\left(M_{0}\right) \cap M_{E} \leftrightarrow \operatorname{Ex}\left(\Lambda_{E}\right) \subset \mathbb{R}^{n} .
$$

We conclude this section with the case where $E$ consists of one point (cf. [2]). If $E=\{z\}$ then such a measure is completely determined by $\mu(z)=c$ and $\mu \in M_{0}$ if and only if

if and only if

$$
0 \leq c \frac{1-|z|^{2}}{|\zeta-z|^{2}} \leq 1 \quad \text { for all } \zeta \in T,
$$

$$
0 \leq c \leq \frac{(1-|z|)^{2}}{1-|z|^{2}}=\frac{1-|z|}{1+|z|} .
$$

Then $c=0$ gives us our well known $\mu=d \theta /(2 \pi)$ and $c=(1-|z|) /(1+|z|)$ gives us an extreme $\mu$ with support $T \cup\{z\}$.

3. Two-point interior supports. Suppose that $E=\left\{a_{1}, a_{2}\right\} \subset D \backslash\{0\}$. Without loss of generality we will assume that $a_{1}=x$ and $a_{2}=y e^{i \alpha}$, $0<x, y<1,0 \leq \alpha \leq \pi$. In the previous section $M_{E}$ was identified with

$$
\Lambda=\Lambda_{E}=\left\{c \in \mathbb{R}_{\geq 0}^{2}:\langle c, p(\theta)\rangle \leq 1 \forall \theta \in[0,2 \pi)\right\} .
$$

In view of Lemma 2.4 the function $\langle c, p(\theta)\rangle$ assumes its maximum on the interval $S=[0, \alpha]$. It follows that

$$
\Lambda=\left\{c \in \mathbb{R}_{\geq 0}^{2}: \forall \theta \in S\langle c, p(\theta)\rangle \leq 1\right\} .
$$

Let

$$
A(\theta)=\left(\begin{array}{ll}
p_{1}(\theta) & p_{2}(\theta) \\
p_{1}^{\prime}(\theta) & p_{2}^{\prime}(\theta)
\end{array}\right)
$$


Then

$$
\operatorname{det} A(\theta)=\frac{2\left(1-x^{2}\right)^{2}\left(1-y^{2}\right)^{2}}{|\zeta-x|^{2}\left|\zeta-a_{2}\right|^{2}}\left(\frac{y \sin (\alpha-\theta)}{\left|\zeta-a_{2}\right|^{2}}+\frac{x \sin \theta}{|\zeta-x|^{2}}\right)
$$

with $\zeta=e^{i \theta}$. For $\theta$ in the interior of $S$, (5) is positive, while if $a_{2} \notin \mathbb{R}$, i.e. $\alpha \neq 0, \pi$, then (5) is positive on all of $S$. Thus a real-analytic map $\gamma(\theta)$ can be defined on the interior of $S$ (and if $a_{2} \notin \mathbb{R}$ even on a neighborhood of $S$ ) by solving

$$
A(\theta) \gamma(\theta)=\left(\begin{array}{l}
1 \\
0
\end{array}\right)
$$

For points where $\gamma^{\prime}(\theta) \neq 0$ the map $\gamma$ defines a smooth curve. We will identify $\gamma$ with its image in $\mathbb{R}^{2}$. In fact, $\gamma$ describes the envelope of the family of lines

$$
l_{\theta}=\left\{c \in \mathbb{R}_{\geq 0}^{2} \mid\langle c, p(\theta)\rangle=1\right\} .
$$

Thus if $\gamma^{\prime}(\theta) \neq 0$, then $l_{\theta}$ is the tangent to $\gamma$ at $\gamma(\theta)$.

From (4) it is clear that boundary points $\left(c_{1}, c_{2}\right) \in \mathbb{R}_{>0}^{2}$ of $\Lambda$ are contained in $\gamma$.

Lemma 3.1. The derivative of $\gamma$ is given by

$$
\gamma_{i}^{\prime}(\theta)=\frac{(-1)^{i} p_{3-i}(\theta) \operatorname{det} A^{\prime}}{\operatorname{det} A^{2}} .
$$

If $0<\alpha<\pi$ then

(a) $\gamma^{\prime}$ has no zeros on $S$, or

(b) $\gamma^{\prime}$ has precisely two zeros on $S$ (counting multiplicity).

Moreover, $\gamma$ is strictly concave where $\gamma_{1}^{\prime}<0$ and strictly convex where $\gamma_{1}^{\prime}>0$. If $\gamma_{1}^{\prime} \leq 0$ then $\gamma$ is a concave curve. Otherwise there exist $0<s<$ $\theta_{1}<\theta_{2}<t<\alpha$ such that $\gamma$ is concave on $\left[0, \theta_{1}\right) \cup\left(\theta_{2}, \alpha\right]$ and convex on $\left(\theta_{1}, \theta_{2}\right)$, while $\gamma(s)=\gamma(t)$.

Pr o of. Differentiating (6), we obtain the following system for $\gamma$ and $\gamma^{\prime}$ :

$$
\left(\begin{array}{cc}
A(\theta) & 0 \\
A^{\prime}(\theta) & A(\theta)
\end{array}\right)\left(\begin{array}{c}
\gamma(\theta) \\
\gamma^{\prime}(\theta)
\end{array}\right)=\left(\begin{array}{l}
1 \\
0 \\
0 \\
0
\end{array}\right)
$$

From Cramer's rule we obtain the expression for $\gamma_{i}^{\prime}$.

We now determine the number of zeros of $\operatorname{det} A^{\prime}$. After some computations we find

$$
\begin{aligned}
\operatorname{det} A^{\prime}= & \frac{4 x y}{|\zeta-x|^{4}\left|\zeta-a_{2}\right|^{4}} \\
& \times\left[\sin \alpha-4 \sin \theta \sin (\alpha-\theta)\left(\frac{y \sin (\alpha-\theta)}{\left|\zeta-a_{2}\right|^{2}}+\frac{x \sin \theta}{|\zeta-x|^{2}}\right)\right] .
\end{aligned}
$$


As the other factors are positive, we only have to study the number of zeros of the factor [...] of $(10)$. Let $u=2 x /\left(1+x^{2}\right) \in(0,1), v=2 y /\left(1+y^{2}\right) \in(0,1)$. Multiply [...] by its denominators and call the result $\psi=\psi_{u, v, a}$. Then

$$
\begin{aligned}
\psi(\theta)= & \sin \alpha-u\left[\sin \alpha \cos \theta+2 \sin ^{2} \theta \sin (\alpha-\theta)\right] \\
& -v\left[\sin \alpha \cos (\alpha-\theta)+2 \sin \theta \sin ^{2}(\alpha-\theta)\right] \\
& +u v \sin \alpha[\cos \theta \cos (\alpha-\theta)+2 \sin \theta \sin (\alpha-\theta)] .
\end{aligned}
$$

Inspection shows that $\psi(0)>0, \psi(\alpha)>0$. Furthermore, $\psi^{\prime}$ is given by

$$
3 \sin (\alpha-2 \theta)[-u \sin \theta-v \sin (\alpha-\theta)+u v \sin \alpha] .
$$

The first factor has one zero at $\theta=\alpha / 2$, the final factor is convex and easily seen to be negative. So $\psi^{\prime}$ has one zero on $S$. The conclusion is that $\psi$ and therefore $\operatorname{det} A^{\prime}$ both have on $S$ either none or two zeros, counting multiplicity. We compute the slope of the tangent $l_{\theta}$ to $\gamma$ at $\gamma_{\theta}$. It equals

$$
\text { slope } l_{\theta}=\gamma_{2}^{\prime}(\theta) / \gamma_{1}^{\prime}(\theta)=-p_{1}(\theta) / p_{2}(\theta) \text {. }
$$

We see that slope $l_{\theta}$ is negative and strictly increasing on $S$. If we keep in mind that $\gamma_{1}^{\prime}(\theta)$ is negative for $\theta \in S$ close enough to 0 or $\alpha$, the lemma follows.

REMARK 3.2. The sign of the second derivative of $\left\langle\gamma\left(\theta_{0}\right), p(\theta)\right\rangle$ at $\theta_{0}$ equals the sign of $\gamma_{1}^{\prime}\left(\theta_{0}\right)$. A maximum (resp. minimum) of $\left\langle\gamma\left(\theta_{0}\right), p(\theta)\right\rangle$ at $\theta_{0}$ corresponds to concavity (convexity) of $\gamma$ at $\theta_{0}$. Indeed, (11) gives $\left\langle\gamma(\theta), p^{\prime \prime}(\theta)\right\rangle=-\left\langle\gamma^{\prime}(\theta), p^{\prime}(\theta)\right\rangle=: g(\theta)$. This gives the matrix equality

$$
A\left(\gamma, \gamma^{\prime}\right)=\left(\begin{array}{cc}
1 & 0 \\
0 & -g(\theta)
\end{array}\right) .
$$

Since $\operatorname{det} A>0$, for every $\theta \in S$ we have $\operatorname{sign} \operatorname{det}\left(\gamma, \gamma^{\prime}\right)=-\operatorname{sign} \gamma_{1}(\theta)^{\prime}=$ $-\operatorname{sign} g(\theta)$.

THEOREM 3.3. We keep the notation as above. If $0<\alpha<\pi$ and $\gamma^{\prime}$ is zero free, then the extreme points of $\Lambda$ are formed by the origin and the smooth curve $\gamma$, while in case $\gamma^{\prime}$ has two zeros on $S$, the extreme points are formed by the origin and a piecewise smooth curve contained in $\gamma$ which connects $((1-x) /(1+x), 0)$ and $(0,(1-y) /(1+y))$ and consists of two smooth parts. If $\alpha=\pi$, then $M_{E}$ is a quadrangle in $\mathbb{R}_{\geq 0}^{2}$ with boundary determined by the two axes and the lines $l_{0}$ and $l_{\pi}$.

Proof. We keep $a_{1}>0$ and assume $0<\alpha<\pi$ first. Let $\partial \Lambda$ denote the intersection of the boundary of $\Lambda$ and $\mathbb{R}_{>0}^{2}$. Observe that by (6), $\gamma(0)=\left(1 / p_{1}(0), 0\right)$ and $\gamma(\alpha)=\left(0,1 / p_{2}(\alpha)\right)$ in conformity with the $|E|=1$ situation; moreover, these are the only points of intersection of $\gamma$ with the positive axes. We know that $\Lambda$ is a convex set in the first quadrant with $\partial \Lambda$ contained in $\gamma$. If $\operatorname{det} A^{\prime} \geq 0$ on $S$ then $\gamma$ is a concave curve in the first quadrant connecting $\gamma(0)$ and $\gamma(\alpha)$ and strictly concave except for maybe 
one point $\theta_{0}$ with $\operatorname{det} A^{\prime}\left(\theta_{0}\right)=0$. We conclude that $\overline{\partial \Lambda}=\gamma$ and $\gamma$ consists of extreme points.

On the other hand, if $\operatorname{det} A^{\prime}$ is negative somewhere, then keeping the notation of Lemma 3.1 we have $\gamma(s)=\gamma(t)$ and

$$
\bar{\partial} \Lambda=\{\gamma(\theta): \theta \in[0, s] \cup[t, \alpha]\} .
$$

This part of $\gamma$ is strictly concave, so again all points of $\overline{\partial \Lambda}=\gamma$ are extreme. If $\alpha=\pi$ then it is clear that $\langle c, p(\theta)\rangle$ has extreme points at $\theta=0, \pi$. Because of the remark after 3.1, $\gamma$ is convex and so, by symmetry, a stationary point $\theta \notin\{0, \pi\}$ of $\langle c, p(\theta)\rangle$ must be a minimum. It follows that at $0, \pi$, the function $\langle c, p(\theta)\rangle$ has two maxima. They must both be $\leq 1$, so $M_{E}$ is indeed described by $\left\{c \in \mathbb{R}_{\geq 0}: l_{0}(c) \leq 1, l_{\pi}(c) \leq 1\right\}$.

The next theorem will show that both cases of Theorem 3.3 can occur.

Definition 3.4. Let $a_{1}, a_{2} \in D \backslash\{0\}$. The pair $\left\{a_{1}, a_{2}\right\}$ is called good if $a_{1}, a_{2}$ are positively dependent or if they are not collinear and $\operatorname{det}(\dot{A}) \geq 0$ on $S$, bad otherwise.

TheOrem 3.5. (a) (Noncollinear) bad pairs exist.

(b) If $\left(a_{1}, a_{2}\right)$ is good then $\left(\lambda a_{1}, \mu a_{2}\right)$ is good for $\lambda, \mu \in(0,1]$.

(c) For every $\alpha \in(0, \pi)$ there is a constant $0<\delta<1$ such that all pairs $\left\{a_{1}, a_{2}\right\}$ in a sector of aperture $\alpha$ and radius $\delta$ are good. Hence (nontrivial) good pairs exist.

Proof. We may assume $a_{1}=x>0, a_{2}=y e^{i \alpha}, 0<\alpha<\pi$.

(a) Suppose that all pairs were good; then (10) would be nonnegative for all $0 \leq \theta \leq \alpha, 0<x, y<1$. Then this is also valid for $x=1=y, \theta=\alpha / 2$ by continuity. Substituting in (11) gives, however,

$$
\psi_{1,1, \alpha}(\alpha / 2)=\sin \alpha-4 \sin (\alpha / 2)+\sin (\alpha)\left(1+\sin ^{2}(\alpha / 2)\right),
$$

which is negative if $0<\alpha<\pi$. Therefore, there exist bad pairs.

(b) This follows from (10) since the factor [...] is decreasing as $x, y$ increase.

(c) This follows from (10) since the terms other than $\sin \alpha$ in [...] can be made arbitrarily small.

4. Finite interior support. In case $|E| \geq 3$ we have only fragmentary results.

Definition 4.1. A finite subset $\left\{a_{1}, \ldots, a_{n}\right\}$ of $D$ is called good if every pair $\left\{a_{i}, a_{j}\right\}, 1 \leq i<j \leq n$, is good.

One might wonder if every finite set in $D$ that is not contained in an interval $\left[0, e^{i \theta}\right]$ (cf. Lemma 2.6) is full support for some extreme measure $\mu \in M_{0}$. The answer is no, as we will now see. 
Theorem 4.2. Let $n \geq 3$ and let $E=\left\{a_{1}, \ldots, a_{n}\right\} \subset D \backslash\{0\}$ be good and suppose $E$ is contained in a sector of aperture $\leq \pi$. Then there is no extreme $\mu \in M_{0}$ with $\operatorname{supp} \mu_{0}=E$.

Proof. Let $\mu \in M_{E}$ be extreme. Put $c=c(\mu)$ as introduced in 2.7 and suppose $c_{i}>0$ for all $i$. Let $\theta_{i}=\arg a_{i}$. We may assume that $0=\theta_{1} \leq \theta_{2} \leq$ $\ldots \leq \theta_{n}<\pi$. Using Lemma 2.4 one easily sees that $\langle c, p(\theta)\rangle$ assumes its maximum only on the arc $\left[0, \theta_{n}\right]$. Moreover, since $\mu$ is a boundary point of $M_{0}$ this maximum equals 1. Also, if $\langle c, p(\theta)\rangle=1$ for $c \in \Lambda_{E}$, then $\left\langle c, p^{\prime}(\theta)\right\rangle=0$ and $\left\langle c, p^{\prime \prime}(\theta)\right\rangle \leq 0$.

Let $V_{\theta}$ be the part of the hyperplane $\langle x, p(\theta)\rangle=1,\left\langle x, p^{\prime}(\theta)\right\rangle=0$ defined by $V_{\theta} \subset \mathbb{R}_{\geq 0}^{n}$. It is clear that there exists a $\theta \in\left[0, \theta_{n}\right]$ such that $c \in V_{\theta}$. If we are able to show that $V_{\theta} \subset \Lambda_{E}$, then we find that $c \in \operatorname{Ex}\left(\Lambda_{E}\right) \Rightarrow c \in$ $\operatorname{Ex}\left(V_{\theta}\right)$, which implies that at least one of the $c_{i}$ vanishes. This leads to a contradiction. The following lemma complets the proof.

Lemma 4.3. $V_{\theta} \subset \Lambda_{E}$ if $\zeta \in\left[0, \theta_{n}\right]$.

Pro of. For $n=2$ this follows from the previous theory by distinguishing two cases: If $\theta_{2}=0$, then $\theta=0$, and $p^{\prime}(0)=0$ so $\left.V_{0}=\mathbb{R}_{\geq 0}^{2} \cap\langle c, p(0)\rangle=1\right\}$ $\subset \Lambda_{E}$. In the second case we have $V_{\theta}=\{\gamma(\theta)\} \subset \Lambda_{E}$ because every pair is good by hypothesis. We will proceed by induction, supposing $n \geq 3$. Note that $V_{\theta} \subset \Lambda_{E} \Leftrightarrow \operatorname{Ex}\left(V_{\theta}\right) \subset \Lambda_{E}$.

Let $q$ be an extreme point of $V_{\theta}$. Then $q_{k}=0$ for a certain $1 \leq k \leq n$. We can apply the induction hypothesis as soon as we know that $\theta$ is in the interval determined by the remaining $\theta_{j}(j \neq k)$. This is certainly the case if $1<k<n$. We next deal only with $k=n$, observing that $k=0$ can be treated similarly.

Assume that $q_{n}=0$. In view of Lemma 2.4, $q_{j} p_{j}(\theta)$ is decreasing on $\left(\theta_{j}, \pi+\theta_{j}\right)$, hence $\langle q, p(\theta)\rangle=\sum_{j=0}^{n-1} q_{j} p_{j}(\theta)$ is decreasing on $\left(\theta_{n-1}, \pi\right]$. Therefore $\left\langle q, p^{\prime}(\varphi)\right\rangle \neq 0$ for $\varphi \in\left(\theta_{n-1}, \pi\right]$. Thus $q \in V_{\theta} \Rightarrow \theta \in\left[0, \theta_{n-1}\right]$.

On the other hand, there may also be extreme points supported on all of $E$, if $|E|=3$.

Theorem 4.4. Let $E=\left\{a_{1}, a_{2}, a_{3}\right\} \subset D \backslash\{0\}$ with 0 in the interior of $\mathrm{CH}(E)$. Then there exists an extreme $\mu \in M_{0}$ with $\operatorname{supp}(\mu)=T \cup E$.

Proof. We may assume that $\arg a_{1}=0<\arg a_{2}<\arg a_{3}<2 \pi$. Let $F$ be the orthogonal complement of $p(0)$ and $p^{\prime}(0)$ in $\mathbb{R}^{3}$. Then $F$ is one-dimensional, spanned by a single vector $f$. Because $p_{1}^{\prime}(0)=0$ and $p_{2}^{\prime}(0) p_{3}^{\prime}(0)<0$, we may assume that $f_{2}, f_{3}>0$ and, consequently, $f_{1}<0$.

Define

$$
\widetilde{c}=\left(\frac{1-a_{1}}{1+a_{1}}, 0,0\right)
$$


the extreme point of $\Lambda_{E}$ on the positive $c_{1}$ axis. Let $c_{\lambda}=\widetilde{c}+\lambda f$ for $\lambda \geq 0$. Notice that all $c \in \mathbb{R}^{3}$ with the property that $\langle c, p(0)\rangle=1,\left\langle c, p^{\prime}(0)\right\rangle=0$ are of the form $c_{\lambda}$. Now we have

$$
c_{\lambda} \in \Lambda_{E} \Leftrightarrow \forall \theta \in[0,2 \pi) \lambda\langle f, p(\theta)\rangle \leq 1-\langle\widetilde{c}, p(\theta)\rangle .
$$

Observe that the right-hand side of the second inequality is positive on $(0,2 \pi)$ and has a double zero at $\theta=0$. Its left-hand side also has (at least) a double zero at $\theta=0$. Thus for sufficiently small but positive $\lambda$ the last inequality is satisfied and $c_{\lambda}$ belongs to $\Lambda_{E}$. If we let $\lambda$ increase, then $c_{\lambda, 1}$ decreases while $c_{\lambda, 2}, c_{\lambda, 3}$ increase. Let $\lambda_{1}$ be the largest value such that $c_{\lambda_{1}} \in$ $\Lambda_{E}$. Then all coordinates of $c_{\lambda_{1}}$ are positive. Otherwise, $c_{\lambda_{1}, 1}$ would be 0 . We show that that this would imply $c_{\lambda_{1}} \notin \Lambda_{E}$. Indeed, $\left\langle c_{\lambda_{1}}, p(0)\right\rangle=1$, but the maximum of $\left\langle c_{\lambda_{1}}, p(\theta)\right\rangle$ is attained on $\left(\arg a_{2}, \arg a_{3}\right)$, which does not contain 0 because $\arg a_{3}-\arg a_{2}<\pi$ by the convexity assumption. The point $c_{\lambda_{1}}$ is an extreme point of $\Lambda_{E}$. If $c_{\lambda_{1}}$ could be written as a convex combination of $d, e \in \Lambda_{E}$, then $d, e$ would be of the form $c_{\lambda}$ because of the behavior at 0 and this implies that $d=e=c_{\lambda_{1}}$.

REMARK 4.5. The preceding theorem is based on the fact that there is a $\theta$ such that the half-line $V_{\theta}$, introduced in the proof of Theorem 4.2, has a segment in common with $\Lambda_{E}$ such that one of the endpoints is in $\mathbb{R}_{>0}^{3}$. This happens precisely in the following situation. Let $E_{i}=E \backslash\left\{a_{i}\right\}$. Theorem 3.3 describes the extreme points of $\Lambda_{E_{i}}$ as points of the form $\gamma_{i}(\theta), \theta \in A_{i} \subset$ $[0,2 \pi)$. If we can choose $\theta$ in $A_{i}$ but outside the other $A_{j}$ 's, then $V_{\theta}$ has the required property. Such a choice of $\theta$ is certainly possible if all 3 pairs are bad.

5. Exposed points of $M_{0}$. Recall that if $p \in C$ is a boundary point of a convex set $C$ in some topological vector space, then there exists a supporting hyperplane at $p$. If the hyperplane $H$ can be chosen with $H \cap C=\{p\}$ then $p$ is called an exposed point of $C$. In our case the vector space is $M$, the space of complex Borel measures on $\bar{D}$ endowed with the weak* topology, and hyperplanes are sets of the form $H=\left\{\mu: \int f d \mu=c\right\}$ where $c \in \mathbb{C}$ and $f \in C(\bar{D}) \backslash\{0\}$.

Again we consider relatively closed subsets $E$ of $D$ and the subsets $M_{E}$ of $M_{0}$ of representing measures defined in the introduction.

Let $X$ be the closure of the convex hull of $\{P(\zeta)|| \zeta \mid>1\} \cup\{f \in C(\bar{D}) \mid$ $f \leq 0\}$ in $C(\bar{D})$. Introduce $\mathcal{M}_{0}=\{\mu \in M \mid \mu(T)=0,\langle\mu, x\rangle \leq 1, x \in X\}$ and its weak* closure is $\overline{\mathcal{M}}=\{\mu \in \mathcal{M} \mid\langle\mu, x\rangle \leq 1, x \in X\}$.

Lemma 5.1. Suppose that $h \in C(\bar{D})$ has the property that $\langle\mu, h\rangle \leq 1$ for all $\mu \in \mathcal{M}_{0}$. Then $h \in X$. 
Pr o of. This is a simple application of the Hahn-Banach theorem. $X$ is a closed convex neighborhood of 0 . If $h \notin X$, then the Minkowski functional $p$ of $X$ is larger than 1 at $h$. Its restriction to the half-line $\{t h \mid t>0\}$ can be extended to a linear functional $\mu \in M$ that is bounded by $p$. Then $\mu \in \overline{\mathcal{M}}$ and $\langle\mu, h\rangle>1$, a contradiction.

Because $\mathcal{M}_{0}$ is starshaped with respect to 0 (i.e. $\mu \in \mathcal{M}_{0}$ implies $t \mu \in$ $\mathcal{M}_{0}$ for $\left.0 \leq t \leq 1\right)$ a supporting hyperplane for a nonzero measure $\mu \in \mathcal{M}_{0}$ cannot pass through 0 . We can thus assume that $c=1$ and $H$ is of the form $\langle\mu, f\rangle=1, f \in C(\bar{D})$, and $\langle\nu, f\rangle \leq 1$ for $\nu \in \mathcal{M}_{0}$. We call $f$ the supporting functional.

Lemma 5.2. Suppose $\mu \in \mathcal{M}_{0}$ is a (non-zero) boundary point with supporting functional $h \in C(\bar{D})$. Then there exists a probability measure $\sigma$ such that $h \leq P[\sigma]$ with equality on $D \cap \operatorname{supp} \mu$ and $\sigma$ is concentrated on $\{\zeta \in T \mid\langle\mu, P(\zeta)\rangle=1\}$.

P r o o f. By Lemma 5.1 we see that $h$ is a uniform limit on $\bar{D}$ of continuous functions $G_{N}$, where $G_{N}=H_{N}-f_{N}$ with $f_{N} \in C(\bar{D})$ nonnegative and

$$
H_{N}(z)=\sum_{j=1}^{M} c_{j} P\left(\zeta_{j}, z\right)
$$

with $c_{j}>0, \sum c_{j} \leq 1,\left|\zeta_{j}\right|>1$ and all depending on $N$. Observe that $H_{N}$ are positive harmonic functions on $\bar{D}$ with

$$
H_{N}(0)=\int_{T} H_{N}\left(e^{i \theta}\right) \frac{d \theta}{2 \pi} \leq 1 .
$$

After taking a subsequence if necessary, we may assume that $H_{N}$ converges weak* to some positive measure $\sigma$ on $T$. The harmonic functions $H_{N}$ will then converge uniformly on compact sets in $D$ to a continuous function. Uniform convergence of the $G_{N}$ then implies that $f_{N}$ converges weak* to some nonnegative measure on $\bar{D}$ and uniformly on compact sets to some continuous $f \geq 0$ on $D$. We have

$$
\begin{aligned}
1 & =\int_{D} \lim _{N \rightarrow \infty} G_{N} d \mu=\lim _{N \rightarrow \infty}\left(\int H_{N} d \mu-\int f_{N} d \mu\right) \\
& \leq \liminf _{N \rightarrow \infty}\left(H_{N}(0)-\int f_{N} d \mu\right) \\
& \leq \lim _{N \rightarrow \infty} H_{N}(0)-\limsup _{N \rightarrow \infty} \int f_{N} d \mu \leq 1 .
\end{aligned}
$$

We conclude that $H_{N}(0) \rightarrow 1$, which implies $|\sigma|=1$, and $f_{N} \rightarrow 0$ in $L^{1}(\mu)$ and uniformly on compact sets in $D \cap \operatorname{supp} \mu$ as $N \rightarrow \infty$. It also follows that $h \leq \lim H_{N}$ as measures on $\bar{D}$ with equality at least on $D \cap \operatorname{supp} \mu$. 
Now recall that $\langle\mu, P(\zeta)\rangle$ is a nonnegative lower semicontinuous function $\leq 1$ which gives meaning to $\int\langle\mu, P(\zeta)\rangle d \sigma$. Using the fact that $h=P[\sigma]$ on $D \cap \operatorname{supp} \mu$ we obtain

$$
1=\int_{D} P[\sigma] d \mu=\int_{T}\langle\mu, P(\zeta)\rangle d \sigma .
$$

From this we infer that $\sigma$ is concentrated on $\{\zeta \in T \mid\langle\mu, P(\zeta)\rangle=1\}$.

Lemma 5.3. If $\mu=\mu_{0}+\mu_{1}$ is an exposed point of $M_{0}$ with $\mu_{0} \neq 0$, then $\mu_{0}$ is an exposed point of $\mathcal{M}_{0}$. The exposing functional can be chosen to be a continuous function $f$ on $\bar{D}$ such that $f=0$ on $T$ and $f=P[\sigma]$ on $D \cap \operatorname{supp} \mu_{0}$ for some probability measure $\sigma$ concentrated on $\{\zeta \in T \mid$ $\langle\mu, P(\zeta)\rangle=1\}$.

Proof. Let $h$ be an exposing functional for $\mu$. Then

$$
\langle\mu, P[h]\rangle=\left\langle\frac{d \theta}{2 \pi}, P[h]\right\rangle=\left\langle\frac{d \theta}{2 \pi}, h\right\rangle<\langle\mu, h\rangle .
$$

Set $c=1 /\langle\mu, h-P[h]\rangle$ and

$$
f(z)=c(h-P[h])(z) .
$$

Then for $\nu \neq \mu$ in $M_{0}$,

$$
\begin{aligned}
\langle\nu, f\rangle & =c(\langle\nu, h\rangle-\langle\nu, P[h]\rangle)=c(\langle\nu, h\rangle-P[h](0)) \\
& <c(\langle\mu, h\rangle-\langle\mu, P[h]\rangle)=\langle\mu, f\rangle .
\end{aligned}
$$

Thus $\langle\mu, f\rangle=1, f$ is an exposing functional, and $\left.f\right|_{T}=0$. Now

$$
\left\langle\mu_{0}, f\right\rangle=\langle\mu, f\rangle>\langle\nu, f\rangle=\left\langle\nu_{0}, f\right\rangle
$$

for all $\nu_{0} \neq \mu_{0}$ in $\mathcal{M}_{0}$. This shows that $\mu_{0}$ is exposed in $\mathcal{M}_{0}$ and the final statements follow from Lemma 5.2.

Let $A=\left\{a_{n}\right\}_{n}$ be a sequence in $D$ without interior limit points and with the property that almost every point of $T$ is a nontangential limit point of $A$. Brown, Shields and Zeller showed that there exist representing measures $\mu$ with $\mu(A)=1$ (see [1]). Let us call such representing measures $B S Z$ measures. In [2] it was shown that there exist BSZ measures that are extreme in $M_{0}$. However:

Proposition 5.4. No BSZ measure is exposed in $M_{0}$, nor in $\mathcal{M}_{0}$.

P r o of. To reach a contradiction, suppose that $\mu$ is a BSZ measure with mass on the sequence $A$ that is exposed in $M_{0}$. Then it would be exposed in $\mathcal{M}_{0}$ by the previous lemma. If $\mu$ is exposed in $\mathcal{M}_{0}$, then the exposing functional may be assumed of the form $h=P[\sigma]$ on $a$. On a small enough tail of $A$ there exists a BSZ measure $\mu^{\prime}$ not equal to $\mu$. However, $\left\langle h, \mu^{\prime}\right\rangle=$ $\left\langle P[\sigma], \mu^{\prime}\right\rangle=1$, contradicting $h$ being the exposing functional. 
REMARK 5.5. A little more generally we may consider a closed subarc $J$ of $T$ of length $\leq \pi$ and a sequence $A$ in $D$ without limit points in $\bar{D} \backslash J$ and such that almost every point of $J$ is a nontangential limit point of a subsequence of $A$. Then one can see that the proof of [1] gives representing measures $\mu$ such that $\mu(J)=0$ and $\mu(a \cup T)=1$. If $A$ is contained in the sector determined by $J$, then $\mu_{0}$ is real-analytic and zero free on $T \backslash J$. The proof of Proposition 5.4 applies (with the note that $l=\sigma$ on $T \backslash J$ ) and shows that such measures cannot be exposed in $M_{0}$.

We will now turn to the case where $E$ is finite and study $M_{E}$ and the associated subsets $\Lambda=\Lambda_{E}$ of $\mathbb{R}^{n}$. Izzo proved the following Theorem 5.8 for the case where $E$ consists of one point (cf. [5]).

Proposition 5.6. Let $E$ be finite in $D$ and let $\nu_{0}$ be a positive measure on $E$. The following are equivalent:

(i) $\nu_{0}$ is an exposed point of $\Lambda_{E}$.

(ii) $\nu_{0}$ is an exposed point of $\mathcal{M}_{0}$.

(iii) $\nu_{0}+\left(1 /(2 \pi)-P_{\nu_{0}}\right) d \theta$ is an exposed point of $M_{0}$.

Proof. (i) $\Rightarrow$ (iii). The methods of Lemmas $5.1,5.2$ readily imply that the exposing functional $h$ on $E$ must be of the form $P[\sigma]$, with $\operatorname{supp} \sigma \subset$ $\left\{\zeta \mid P\left(\zeta, \nu_{0}\right)=1\right\}$. This is a finite set, therefore $\sigma$ is singular and $P[\sigma]=0$ a.e. on $T$. We now choose $\widetilde{h}$ in $C(\bar{D})$ such that $\widetilde{h} \leq P[\sigma]$ on $D$ with equality only on $E$, while $\widetilde{h}=0$ on $T$. We claim that $\widetilde{h}$ exposes $\nu$ in $M_{0}$. Indeed, for $\mu \in M_{0}$,

$$
\langle\widetilde{h}, \mu\rangle=\left\langle\widetilde{h}, \mu_{0}\right\rangle \leq\left\langle P[\sigma], \mu_{0}\right\rangle \leq 1 .
$$

To have equality in the first inequality, we must have supp $\mu_{0} \subset E$. But then equality in the last inequality can only occur if $\mu_{0}=\nu_{0}$. It follows that $\mu=\nu$. Lemma 5.3 states that (iii) $\Rightarrow($ ii); finally, (ii) $\Rightarrow(\mathrm{i})$ is obvious.

Definition 5.7. We will call an extreme point $\mu_{0} \in M_{E}$ regular if $\mu_{1}$ has only zeros of order 2 .

TheOREM 5.8. Suppose that $E$ is finite. Then every regular extreme point of $M_{E}$ is an exposed point of $M_{E}$.

Proof. Let $E=\left\{a_{1}, \ldots, a_{n}\right\} \subset D$. As in the previous section we may identify $M_{E}$ with a convex subset $\Lambda=\Lambda_{E}$ in the positive octant:

$$
\Lambda_{E}=\left\{c \in \mathbb{R}_{\geq 0}^{n} \mid\langle c, p(\theta)\rangle \leq 1 \forall \theta \in[0,2 \pi)\right\} .
$$

The proof is by induction on $n$, starting at $n=3$. For $n=1$ it is clear that an extreme $m_{0}$ is exposed in $M_{E}$, if $n=2$ it follows from the description of the boundary of $M_{E}$ in Theorem 3.3, as a concave curve which does not contain intervals, that every extreme point is exposed in $\Lambda_{E}$ and by Proposition 5.6 also in $M_{E}$. Suppose $c_{0}$ is an extreme point of $\Lambda$. We may 
assume that all components of $c_{0}$ are positive, otherwise we could reduce $n$ and apply Proposition 5.6 to show that such a point is exposed within $M_{0}$, hence also in $M_{E}$. Consider the set

$$
S=\left\{\zeta \in T \mid\left\langle c_{0}, P(\zeta)\right\rangle=1\right\} .
$$

Clearly, $S$ is a finite subset of $T$ since $\left\langle c_{0}, P(\zeta)\right\rangle$ is real-analytic and nonconstant on $T$. Also, $S$ contains at least one point, otherwise $c_{0}$ is not even in the boundary of $M_{E}$. Now let

$$
X_{S}=\left\{c \in \Lambda_{E} \mid\langle c, P(\zeta)\rangle=1, \zeta \in S\right\} .
$$

We claim that $X_{S}=\left\{c_{0}\right\}$. Obviously, $c_{0} \in X_{S}$. If there exists an additional point $c \in X_{S}$, then $X_{S}$ would be a convex subset of $\Lambda_{E}$ that contains the interval $\left[c_{0}, c\right]$ Consider $c_{\varepsilon}=c_{0}+\varepsilon\left(c_{0}-c\right)$, where $\varepsilon$ will be chosen positive. For $\zeta \in S, \zeta=e^{i \theta}$, we have $\left\langle c_{\varepsilon}, p(\theta)\right\rangle=1$ and $\left\langle c_{\varepsilon}, p^{\prime}(\theta)\right\rangle=0$. Moreover, since $c_{0}$ is regular, we have $\left\langle c_{0}, p^{\prime \prime}(\theta)\right\rangle>\delta>0$ on a neighborhood of $S$. Therefore, for $0<\varepsilon<\varepsilon_{0}$ sufficiently small, $\left\langle c_{\varepsilon}, p^{\prime \prime}(\theta)\right\rangle>\delta / 2$ for $\zeta=e^{i \theta}$ in a smaller neighborhood $V$ only depending on $\varepsilon_{0}$. Thus on $V$ we have $\left\langle c_{\varepsilon}, P(\zeta)\right\rangle<1$. On $T \backslash V$ we have $\left\langle c_{0}, P(\zeta)\right\rangle \leq 1-\eta$, hence for $\varepsilon$ sufficiently small we have $\left\langle c_{\varepsilon}, P(\zeta)\right\rangle<1$ on $T \backslash V$. The conclusion is that $c_{\varepsilon} \in \Lambda_{E}$. We can now write $c_{0}$ as a convex combination of $c_{\varepsilon}$ and $c$, contradicting that $c_{0}$ is extreme. For the exposing functional for $c_{0}$ we may now take

$$
w=\frac{1}{n} \sum_{\zeta \in S} P(\zeta),
$$

where $n=\# S$. We have $\left\langle c_{0}, w\right\rangle=1$ and $\langle c, w\rangle=1$ implies that for all $\zeta \in S,\langle c, P(\zeta)\rangle=1$, hence $c \in X_{S}$, that is, $c=c_{0}$.

REMARK 5.9. What will happen if $c_{0}$ is extreme but not regular? We keep the notation of the previous theorem. It is clear, from the proof and the fact that exposing functionals must be of the form $P[\sigma]$ with $\sigma$ supported in $S$, that $\left\{c_{0}\right\}=X_{S}$ is equivalent to $c_{0}$ being exposed. If $n=2$ this will always be the case, although $c_{0}$ is nonregular precisely in the borderline case when $\gamma^{\prime}$ has one zero with multiplicity 2 (cf. Lemma 3.1). On the other hand, a little experimenting with Maple gives the following example of an extreme point which is not exposed. Take three points: $a_{1}=1 / 10, a_{2}=\frac{3}{20} e^{i \pi / 3}, a_{3}=\bar{a}_{2}$. Put weight $c_{1}=1710234 / 42508549$ at $a_{1}$ and equal weights $c_{2}=c_{3}=1$ at $a_{2}$ and $a_{3}$. We obtain a point in the boundary of $\Lambda=\Lambda_{\left\{a_{1}, a_{2}, a_{3}\right\}}$ by multiplying by a suitable positive constant. Call this point $c$. Then the Poisson transform $\langle c, p(\theta)\rangle$ will have its only maximum at $\theta=0$ with the property that the first three $\theta$-derivatives vanish at $\theta=0$. The point $c$ is an extreme point of $\Lambda$. It is, however, not exposed, because $X_{S}$ is the convex hull of $c$ and $\left(\left(1-a_{1}\right) /\left(1+a_{1}\right), 0,0\right)$. 


\section{References}

[1] L. Brown, A. Shields and K. Zeller, On absolutely convergent exponential sums, Trans. Amer. Math. Soc. 96 (1960), 162-183.

[2] R. Brummelhuis and J. Wiegerinck, Representing measures for the disc algebra and the ball algebra, Ann. Polon. Math. 55 (1991), 19-35.

[3] T. Gamelin, Uniform Algebras, Prentice-Hall, Englewood Cliffs, N.J., 1969.

[4] J. B. Garnett, Bounded Analytic Functions, Academic Press, New York, 1981.

[5] A. J. Izzo, Exposed points in the set of representing measures for the disc algebra, Ann. Polon. Math. 61 (1995), 59-62.

[6] P. Koosis, Theory of $H^{p}$ Spaces, Cambridge Univ. Press, Cambridge, 1980.

[7] J. Ryff, The support of representing measures for the disc algebra, in: Function Algebras, F. Birtel (ed.), Scott, Foresman and Co., Glenview, 1966, 112-117.

Mathematical Institute

University of Leiden

P.O. Box 9512

2300 RA Leiden, The Netherlands

E-mail: heinis@wi.leidenuniv.nl
Korteweg-de Vries Institute

University of Amsterdam

Plantage Muidergracht 24

1018 TV Amsterdam, The Netherlands

E-mail: janwieg@wins.uva.nl

Reçu par la Rédaction le 18.6.1998

Révisé le 17.11.1999 